EPJ Web of Conferences 13, 04005 (2011)

DOI: $10.1051 /$ epjconf/20111304005

C Owned by the authors, published by EDP Sciences, 2011

\title{
Charged particles produced in quark and gluon jets in proton-proton collisions
}

\author{
Sona Pochybova ${ }^{1, a}$ \\ 1 MTA KFKI RMKI, Konkoly-Thege Miklós Str. 29-33, H-1121 Budapest, Hungary \\ 2 Eötvös University, Pázmány Péter sétány 1/A, H-1117 Budapest, Hungary
}

\begin{abstract}
The study of particle production inside jets makes it possible to address the fragmentation properties of the leading parton depending on its flavour. We investigate whether different fragmentation properties of quarks and gluons affect identified particle spectra.

We present a systematic study of $K, p$ and $\pi$ production at RHIC, Tevatron and LHC energies. Through the study of various event shapes we can directly access the fragmentation properties of quark and gluon jets. We present MC estimate for the contribution of quark and gluon jets to individual particle species spectra, that can be compared to experimental results and test our current knowledge of the physics behind particle production inside jets. Where possible, we cross-check our results with existing data and further extend the study to higher energies.
\end{abstract}

\section{Introduction}

Jets are produced in hard scatterings of partons of colliding particles. Emerging from the early stages of collisions they are ideal tools to study final states, hadronisation processes and hadron production. Such questions can be addressed through investigation of fragmentation properties of quark and gluon jets in different event shapes (2- or 3- jet) and jet-production channels.

Quarks and gluons carry different color factors. Gluon carries colour factor $\mathrm{C}_{A}=3$ and quark carries colour factor $\mathrm{C}_{F}=4 / 3$

$$
\frac{\mathrm{C}_{A}}{\mathrm{C}_{F}}=\frac{9}{4}
$$

The colour factors are proportional to the probability that a parton radiates a soft gluon. Colour factor of a gluon is more than two times larger than the one of a quark in asymptotic limit of $Q^{2} \rightarrow \infty$. This means, that gluons branch more easily and form higher multiplicity jets. Thus, gluon jets are expected to be broader with softer fragmentation function than quark jets [1], [2]. Apart from overall differences, the individual partons are expected to contribute differently to the yields of specific hadrons. Namely, gluons enhance baryons in an event, whereas quarks contribute mainly to meson production [3].

In the following, we show how gluon contribution to hadron spectra changes with collision energy, moreover, that this contribution is dominant for protons. In the case of 2- and 3-jet events, we show that additional hard gluon radiation has an effect of $20 \%-40 \%$ to the relative proton production. The presented MC analysis is an extension of

\footnotetext{
a e-mail: sona.pochybova@cern.ch
}

multiple jet studies [4]-[8], with the perspective of implication in the future experiments at LHC, which have good particle identification capabilities up to high momenta.

The data were simulated using PYTHIA event generator [9] with the settings of Perugia-0 tune [10]. Three types of data-sets, were created for each collision energy - $200 \mathrm{GeV}, 1800 \mathrm{GeV}$ and $7 \mathrm{TeV}$ including pure gluon (GG), pure quark (QQ) and mixed (QG) jet event production. Every sample contains 300000 events. Separating the production channels, we were able to see the parton-type effect on single hadron spectra. Additionally, as a reference, three respective minimum bias (MB) samples were generated, each containing $1 \mathrm{M}$ events.

In order to study particle production in different event shapes, we selected 2- and 3-jet-like events based on the so called thrust variable, $T$ [9]. Events with $T$ smaller than 0.9 were treated as 3 -jet-like, events with higher values as 2-jet-like. This separation was earlier proposed for multijet analysis at LEP [11].The effects of GG, QQ and QG production channels were studied via $p+\bar{p} / K^{+}+K^{-}(\mathrm{p} / \mathrm{K})$ and $p+\bar{p} / \pi^{+}+\pi^{-}(\mathrm{p} / \pi)$ ratios.

\section{$2 \mathrm{p} / \pi$ and $\mathrm{p} / \mathrm{K}$ ratios in selected production channels}

At first we examined the separate contributions of individual jet-production channels to the whole spectra (see Table 1 ) and to the $\mathrm{p} / \pi$ and $\mathrm{p} / \mathrm{K}$ ratios (Fig. 2).

In the case of jet sample generation, we have taken into account, that the cross section for jet production varies strongly with jet energy. For this reason, the jet sample was generated in three $p_{T}^{\text {hard }}$ bins $\left(p_{T}^{\text {hard }}=\{15-50 \mathrm{GeV} / \mathrm{c}, 50-\right.$ $100 \mathrm{GeV} / \mathrm{c}, 100 \mathrm{GeV} / \mathrm{c} \leq\})$, where $p_{T}^{\text {hard }}$ is the transverse

This is an Open Access article distributed under the terms of the Creative Commons Attribution-Noncommercial License 3.0, which permits unrestricted use, distribution, and reproduction in any noncommercial medium, provided the original work is properly cited. 

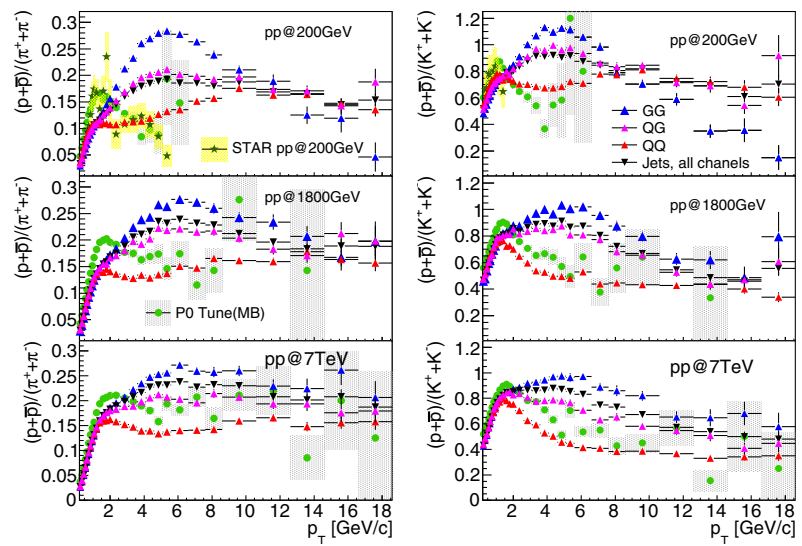

Fig. 1. $p / \pi$ (left panels) and $p / K$ (right panels) for various production channels; GG (blue triangles), QG (magenta triangles), QQ (red triangles) compared to all production channels (black triangles) and MB (green circles). At $\sqrt{s}=200 \mathrm{GeV}$ ratio is compared to STAR data [12]

\begin{tabular}{lrrrrr}
\hline$\sqrt{s}$ & Jet/MB & GG/Jet & QQ/Jet & QG/Jet & 3-/2-Jet \\
\hline $0.2 \mathrm{TeV}$ & $0.1 \%$ & $17.7 \%$ & $27.3 \%$ & $55.0 \%$ & $4.0 \%$ \\
$1.2 \mathrm{TeV}$ & $34.2 \%$ & $49.7 \%$ & $7.6 \%$ & $42.7 \%$ & $11.0 \%$ \\
$7.0 \mathrm{TeV}$ & $95.0 \%$ & $60.0 \%$ & $5.3 \%$ & $34.7 \%$ & $18.0 \%$ \\
\hline
\end{tabular}

Table 1. Integrated fraction of jet events in MB production (second column) and fraction of various production channels contained in a jet production. In the last column; the fraction of 3over 2-jet events.

momentum of the partons in the rest frame of the interaction. After proper cross section scaling, the partial $p_{T}^{\text {hard }}$ samples where merged into one.

From Tab 1 we see, that the fraction of jet events contributing to the MB spectra rises with energy, furthermore, jet sample becomes gluon dominated.

For both $\mathrm{p} / \pi$ and $\mathrm{p} / \mathrm{K}$, in the $2-6 \mathrm{GeV} / \mathrm{c}$ region, the ratio reaches the highest value for GG events. Specifically, $\mathrm{p} / \pi$ ratio reaches values between $\approx 0.25-0.3$ and $\mathrm{p} / \mathrm{K}$ ratio rises up to $\approx 1$. The combined ratio for all channels and the GG ratio get closer with collision energy (Fig. 1), demonstrating the gluon dominance in the sample. This behaviour can be connected to how protons are formed within the popcorn fragmentation model in PYTHIA [9], [13]. Observing the ratios experimentally can contribute to further understanding of particle production mechanisms.

The MB points seem to prefer the region between QG and QQ values, especially in the region above $3 \mathrm{GeV} / \mathrm{c}$, where hard scattering becomes important. The MB ratio rises with collision energy towards $\mathrm{QG}$ value.

As we go from $200 \mathrm{GeV}$ to $7 \mathrm{TeV}$ we are leaving the quark dominated region and entering a gluon dominated one (see Tab. 1). Thus, one would expect the ratio to rise with collison energy, however, this is not the case. Instead, the combined $p / \pi$ ratio stays rather same, whereas the GG value decreases. Such a behaviour may originate in the settings of the used MC tune, that is based on past experimental tests, in which the quark and gluon production channels were not treated separately. This point stressses the importance of studying quark and gluon jets for MC tuning purposes.

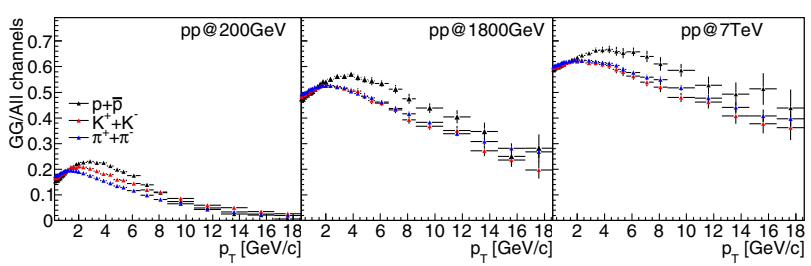

Fig. 2. GG production channel contribution to all production channels for individual particle spectra.

In Fig. 2 we see the GG channel contribution to the jet spectra for individual hadrons as it changes with hadron momentum and collision energy. The contribution weakens with momentum and rises with collision energy (also see Table 1). The highest contribution from the GG channel belongs to protons, which complements Fig. 2.

\subsection{Particle ratios in 2- and 3-jet like events}

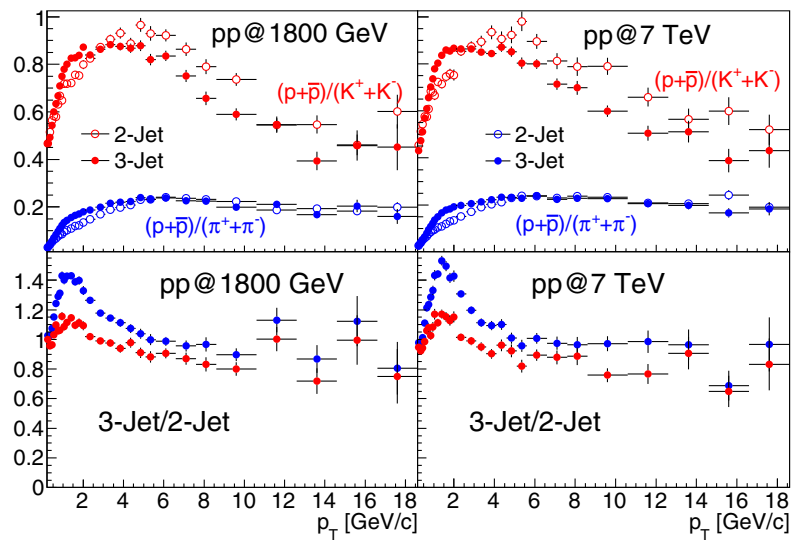

Fig. 3. Top panels: $\mathrm{p} / \pi$ (blue) and $\mathrm{p} / \mathrm{K}$ (red) ratios for all production channels spectrum. Bottom panels: (3-jet ratio)/(2-jet ratio).

In the second part of our investigation, we focused on how the ratios change in the presence of additional hard gluon radiation, i.e. in 3-jet events. This was achieved by distinguishing between the anisotropic and the isotropic event shapes, which correspond to 2- and 3-jet-like events respectively using th $T$ variable.

In Fig. 2.1 we plot the $\mathrm{p} / \mathrm{K}$ and $\mathrm{p} / \pi$ ratios for $2-$ and 3 -jet like events. The hard gluon radiation, present in 3-jet like events, causes an excess in proton spectrum w.r.t pion or kaon. The effect being stronger for $\mathrm{p} / \pi$. This is true for regions below $6 \mathrm{GeV} / \mathrm{c}$, where increase is up to $\approx 40 \%$ for $\mathrm{p} / \pi$ and up to $\approx 20 \%$ for $\mathrm{p} / \mathrm{K}$. The enhancement, caused by hard gluon radiation, in the individual spectra originates, as mentioned in section 2, in the fragmentation model used within PYTHIA. 
Hard gluons causing the effect observed in Fig. 2.1, carry the smallest momentum in the jet-system. Thus, they contribute to particle spectra at lower $p_{T}$. This can explain the vanishing of differences between 2- and 3-jet-like events above $6 \mathrm{GeV} / \mathrm{c}$.

\section{Discussion}

We presented a MC study of hadrons produced in hard scatterings in proton-proton collisions. Through the investigation of $\mathrm{p} / \mathrm{K}$ and $\mathrm{p} / \pi$ ratios in different jet-production channels we were able to see if and how the differences in the ratio values reflect the different fragmentation properties of quarks and gluons. Generally, we observed that gluon contribution to jet spectra rises with energy (see Table 1), and it is highest for protons (Fig. 2, Fig. 2).

When comparing 2- and 3-jet like events, we saw that additional hard gluon radiation, which is present in the 3jet case, causes an enhancement in proton spectrum w.r.t pion (up to $\approx 40 \%$ ) as well as kaon (up to $\approx 20 \%$ ) (Fig. 2.1).

To conclude, the differences in particle production in the individual production channels (GG, QG and QQ) as well as 2- and 3-jet events are present in the mid- $p_{T}$ region $(2-6 \mathrm{GeV} / \mathrm{c})$ and are directly connected to the fragmentation model used in PYTHIA. In this sense, the presented study suggests methods to investigate fragmentation in an experimentally interesting momentum region for this kind of analysis.

\section{References}

1. B. R. Weber, Physica Scripta. 25 (1982) 198-211.

2. R. K. Ellis, W. J. Stirling and B. R. Webber, Camb. Monogr. Part. Phys. Nucl. Phys. Cosmol. 8 (1996) 1.

3. S. Albino, B. A. Kniehl and G. Kramer, Nucl. Phys. B 725 (2005) 181.

4. P. Abreu et al. [DELPHI Collaboration], Z. Phys. C 70 (1996) 179.

5. R. Barate et al. [ALEPH Collaboration], Eur. Phys. J. C 17 (2000) 1.

6. G. Alexander et al. [OPAL Collaboration], Z. Phys. C 69 (1996) 543.

7. A. A. Affolder et al. [CDF Collaboration], Phys. Rev. Lett. 87 (2001) 211804.

8. V. M. Abazov et al. [D0 Collaboration], Phys. Rev. D 65 (2002) 052008.

9. T. Sjostrand, S. Mrenna and P. Z. Skands, JHEP 0605 (2006) 026.

10. Peter Z. Skands [Fermilab] FERMILAB-CONF-09113-T, May 2009.

11. F. Barreiro, S. Brandt and M. Gebser, Nucl. Instrum. Meth. A 240 (1985) 237.

12. John Adams et al. [STAR Collaboration] Phys. Lett. B 637 (2006) 161.

13. B. Andersson, G. Gustafson, G. Ingelman and T. Sjostrand, Phys. Rept. 97 (1983) 31. 